\title{
The Herbst appliance and TMJ morphology: is there an effect?
}

\author{
Does the Herbst appliance have any effect on temporomandibular joint \\ morphology?
}

\begin{abstract}
Popowich K, Nebbe B, Major PW. Effect of Herbst treatment on temporomandibular joint morphology: a systematic literature review. Am J Orthod Dentofac Orthop 2003; 123:388-394
\end{abstract}

Data sources Medline, Best Evidence, the Cochrane Database of Systematic Reviews, Embase, abstracts of related articles and the reference lists of the retrieved articles were searched by hand.

Study selection To be selected, work had to be in the form of controlled studies (minimum-internal control with pre-intervention and post-intervention imaging) of Class II malocclusions treated with the Herbst appliance. Magnetic resonance imaging (MRI) or computerised tomography (CT) scans or tomography (axially or horizontally corrected) had to have been used to image the temporomandibular joint (TMJ). Case reports were excluded.

Data extraction and synthesis Eligibility was determined by two authors and agreement reached by discussion. A qualitative synthesis of data was carried out.

Results Of 80 studies identified, only five met the inclusion criteria. All studies used internal controls with pre-treatment and post-treatment imaging. Four studies used MRI and one used tomograms. The four MRI studies used overlapping patient samples and were not considered to be independent evidence. The MRI studies did not provide conclusive evidence of osseous remodelling or condyle position change. The tomography study demonstrated minor condyle position change. Methodological deficiencies prevented major conclusions regarding disc position.

Conclusions There is a need for randomised controlled studies with serial MRI and tomography to establish short- and longterm effects of Herbst treatment on TMJ osseous and soft tissue morphology.

\section{Commentary}

Functional appliances have been used to treat Class II malocclusions for over a century now. The appliance treatment, sometimes known as dentofacial orthopaedics, has been shown to alter the TMJ and lengthen the mandible. The Herbst appliance is a fixed version of the functional appliance with a high clinical success rate because it is not removable and constantly postures the mandible forwards.

Much of the early evidence obtained from animal histology showed osteogenic changes to the TMJ, but similar studies were never carried out on human subjects for obvious reasons. Thus, the effects of the functional appliance on the TMJ have never been fully understood. In recent years, evidence from well-designed randomised controlled trials on Class II malocclusions have concluded that $70-80 \%$ of changes brought about by functional appliances are dento-alveolar. ${ }^{1,2}$ Because of the difficulty and poor information obtained from diagnostic evaluation of the TMJ, inconclusive results have been drawn from the studies relating to this area of the jaw.

Address for correspondence: Paul W Major, Department of Dentistry, Faculty of Medicine and Dentistry, \#4051B Dentistry/Pharmacy Centre, University of Alberta, Edmonton, Alberta, Canada T6G 2N8. E-mail: major@ualberta.ca
The aim of this systematic review was to determine if Herbst appliance therapy altered TMJ morphology. To obtain accurate evidence, only information from CT, MRI and tomography were selected. Five studies were chosen from a possible 80, of which four were from the same centre. Four main areas of the TMJ were reported on: glenoid fossa remodelling, condyle remodelling, condylar position and temporomandibular disc position.

This review reported that, from the evidence available, there were signs of remodelling to the glenoid fossa and condyle as a result of treatment. No pathological changes were reported to be associated with the condyles 1 year post-treatment, however. With regard to condylar positioning, one study reported no change, whereas another reported a slight change post-treatment, although the evidence from the latter did not show a substantial difference. Finally, the evidence relating to changes to disc position was also inconclusive.

In general, this was a well-designed systematic review. It highlighted the deficiencies associated with functional appliance treatment and its effect on the TMJ. These include the lack of a quantitative method to superimpose the condyles throughout the study period, the variability amongst clinicians in appraising TMJ changes and the selection of controls to compare treatment changes. Furthermore, it emphasised that the number of independent studies present in the literature were lacking. As a result of the findings, it was suggested that more randomised clinical trials are needed. There is a balance to make, though, hinging on the need for clinical knowledge but also the ethical dilemma of withdrawing treatment from a patient and the exposure of high radiation dosages to the growing child.

To conclude, the evidence that the Herbst appliance affects TMJ morphology is inconclusive and more work is required. The question is, how we are going to do this? With the innovations in trial design and technological advances in 3D imaging techniques, we might just find the answers in the not too distant future!

\section{Practice point}

- More research is required to better understand the effects of the Herbst appliance on TMJ morphology.

\section{Chung How Kau}

Dental Health and Biological Sciences, Dental School, University of Wales College of Medicine, Cardiff, Wales, UK

1. O'Brien K, Wright J, Conboy F, et al. Effectiveness of early orthodontic treatment with the twin-block appliance: a multicenter, randomized, controlled trial. Part 1. Dental and skeletal effects. Am J Orthod Dentofac Orthop 2003; 124:234-243.

2. Tulloch JF, Proffit WR, Phillips C. Outcomes in a 2-phase randomized clinical trial of early Class II treatment. Am J Orthod Dentofacial Orthop 2004; 125:657-667.

Evidence-Based Dentistry (2004) 5, 105.

doi:10.1038/sj.ebd.6400271 